\title{
Anticipation in the Synchronization of Chaotic Semiconductor Lasers with Optical Feedback
}

\author{
C. Masoller \\ Instituto de Física, Facultad de Ciencias, Universidad de la República, Igua 4225 Montevideo 11400, Uruguay
}

(Received 13 November 2000)

\begin{abstract}
The synchronization of chatoic semiconductor lasers with optical feedback is studied numerically in a one-way coupling configuration, in which a small amount of the intensity of one laser (master laser) is injected coherently into the other (slave laser). A regime of anticipated synchronization is found, in which the intensity of the slave laser is synchronized to the future chaotic intensity of the master laser. Anticipation is robust to small noise and parameter mismatches, but in this case the synchronization is not complete. It is also shown that anticipated synchronization occurs in coupled time-delay systems, when the coupling has a delay that is less than the delay of the systems.
\end{abstract}

DOI: $10.1103 /$ PhysRevLett.86.2782

PACS numbers: 05.45.Xt, 42.55.Px, 42.65.Sf

The synchronization of chaotic systems in a unidirectional coupling configuration has attracted a lot of attention in recent years, in part due to its potential application to secure communication [1-7]. The idea is to use the chaotic output of a transmitter as a carrier in which a message is encoded (the message must be small enough to be masked by the fluctuations of the chaotic signal). The signal is transmitted to a synchronized chaotic receiver, from which the message can be decoded. Unfortunately, most of the schemes proposed are not as secure as expected. Several studies have shown that when using nonlinear dynamics techniques the message can be unmasked [8,9].

Of significant interest is the synchronization of chaotic semiconductor lasers, since these devices have potential application for high-speed optical communications. One way of inducing chaotic emission in a semiconductor laser is by optical feedback from an external reflector. Optical feedback can destabilize the laser, causing it to enter a regime characterized by high-intensity noise and a very broad linewidth, which has been shown to be a form of chaotic dynamics [10]. Several groups have demonstrated the synchronization of chaotic oscillations, and the possibility of message encoding and decoding [11-17].

In this Letter, the synchronization of two semiconductor lasers with optical feedback is studied numerically. The finite time interval required for the light to travel from one laser (master laser) to the other (slave laser) is taken into account by a retardation time, $\tau_{c}$, in the optical coupling. A regime of anticipated synchronization [18] is found, when the round-trip time of the light in the external cavity, $\tau$, is greater than $\tau_{c}$. In this regime, which occurs for large enough coupling strength and adequate feedback levels, the slave laser anticipates the chaotic output of the master laser by an amount of time given by $\tau-\tau_{c}$. When $\tau<\tau_{c}$, retarded synchronization occurs, with the chaotic emission of the slave laser delayed in time with respect to the emission of the master laser, an amount of time given by $\tau_{c}-\tau$. Retarded synchronization was previously reported by Ahlers et al. [15], based on numerical simulations of a similar model.
To the best of our knowledge, anticipation in the synchronization of chaotic semiconductor lasers has not been reported previously in the literature. Experimentally, the time difference between the waveforms in the master and slave lasers was found to depend only on the value of $\tau_{c}$ $[12,13]$, and the observed synchronization was interpreted to be caused by injection locking of the slave to the master laser. In numerical simulations, anticipation has not been reported, perhaps because, in previous work, attention was focused on the synchronization of distant lasers (such that $\left.\tau_{c}>\tau\right)$.

Anticipating synchronization is a regime that was recently discovered by Voss [18]. In Ref. [18] the author gave analytic and numerical evidence of the occurrence of this regime in a system of two coupled scalar delaydifferential equations, in a one-way delayed coupling configuration. In the coupling setup considered here, which is a generalization of the one considered in [18], the delay time in the coupling is generally different from the delay time of the coupled systems. It could be expected that a different time delay in the coupling would defy synchronization because it changes the structures of the coupled systems, and it is hard to synchronize nonidentical systems. However, it is shown that robust synchronization occurs, such that one system lags in time to the other, with the lag time determined only by the difference between $\tau$ and $\tau_{c}$.

The rate equations for two single-mode semiconductor lasers with optical feedback and optical injection from one laser to the other are [19]

$$
\begin{aligned}
\frac{d E_{i}}{d t}= & k_{i}\left(1+i \alpha_{i}\right)\left[G_{i}-1\right] E_{i}(t)+\gamma_{i} E_{i}(t-\tau) \\
& \times \exp \left[-i \omega_{i} \tau_{i}\right]+\eta E_{1}\left(t-\tau_{c}\right) \\
& \times \exp \left[-i\left(\omega_{1} \tau_{c}+\Delta \omega t\right)\right]+\beta_{i} \xi_{i}(t), \\
& \frac{d N_{i}}{d t}=\frac{j_{i}-N_{i}-G_{i}\left|E_{i}\right|^{2}}{\tau_{n i}} .
\end{aligned}
$$

Here, the indices $i=1$ and $i=2$ label the master and the slave laser, respectively, $E_{i}$ is the slowly varying complex 
field, and $N_{i}$ is the normalized carrier density. The term $\eta E_{1}\left(t-\tau_{c}\right) \exp \left[-i\left(\omega_{1} \tau_{c}+\Delta \omega t\right)\right]$ in (1) exists only for the slave laser, and accounts for the light injected from the master laser. The parameters are as follows: $k_{i}$ is the cavity losses, $\alpha_{i}$ is the linewidth enhancement factor, $G_{i}=N_{i} /\left(1+\epsilon_{i}\left|E_{i}\right|^{2}\right)$ is the optical gain (where $\epsilon_{i}$ is the gain saturation coefficient), $\gamma_{i}$ is the feedback level, $\eta$ is the coupling coefficient, $\omega_{i}$ is the optical frequency without feedback, $\Delta \omega=\omega_{2}-\omega_{1}$ is the frequency detuning between the lasers, $\tau_{i}$ is the round-trip time in the external cavity, and $\tau_{c}$ is the time the light flies from the master to the slave laser. $\xi_{i}(t)$ are independent complex Gaussian white noises, and $\beta_{i}$ measures the noise intensity. $j_{i}$ is the normalized injection current, and $\tau_{n i}$ is the carrier lifetime. The model does not include multiple reflections in the external cavity, and therefore it is valid for weak feedback levels. In spite of the fact that weak optical feedback can cause a single-mode laser to operate chaotically on several of its previously suppressed longitudinal modes, the single-mode rate equations [19] have been successful in describing many of the observed phenomena.

Perfectly synchronized solutions of (1) and (2) exist only if the lasers are identical, noise is neglected, and $\gamma_{1}=$ $\gamma_{2}+\eta$ [15]. In this situation, if $E_{1}$ and $E_{2}$ are related by $E_{1}\left(t-\tau_{c}\right) \exp \left[-i\left(\omega \tau_{c}\right)\right]=E_{2}(t-\tau) \exp [-i(\omega \tau)]$, the equations for the optical fields of the lasers are identical, and completely synchronized solutions are, in principle, possible. In these solutions the output intensities are related by $I_{1}(t)=I_{2}\left(t-\tau+\tau_{c}\right)$ (where $I_{1}=\left|E_{1}\right|^{2}$, $I_{2}=\left|E_{2}\right|^{2}$ ). Depending on the difference $\tau-\tau_{c}$, there is anticipated or retarded synchronization.

Figure 1 shows numerical solutions when there is anticipated synchronization. In Figs. 1(a)-1(c), the master laser operates at about $1 \%$ above threshold, and is subjected to moderately strong feedback. For these parameters the laser exhibits low-frequency fluctuations (LFFs) [20]. The intensity suddenly drops toward zero and then recovers gradually, only to drop out again after a random time interval. The intensity dropouts are actually the envelope of a series of fast, picosecond pulses. In Fig. 1(a) the lasers are identical, and the noise level is zero. For large enough $\eta$, and for $\gamma_{2}=\gamma_{1}-\eta$, the slave laser anticipates by $5 \mathrm{~ns}$ the output of the master laser. The dotted lines plot $I_{1}\left(t+\tau-\tau_{c}\right)-I_{2}(t)$, and prove that, after a transient time, the slave laser is perfectly synchronized to the future chaotic output of the master laser.

The physical origin of this behavior can be understood by looking at the simultaneous turn-on of the master and slave lasers [see Fig. 1(b)]. The lasers emit the first intensity pulse at approximately the same time. The master laser emits a train of pulses at the relaxation-oscillation period, before relaxing to the solitary steady state. This train of pulses interferes with the steady state, when it returns from the external mirror, at time $\tau$ after the emission of the first pulse. A fraction of the master intensity is transmitted to the slave laser, and the train of pulses interferes with the slave laser emission, at time $\tau_{c}$ after the emission of the first pulse. Therefore, if the coupling is strong enough, the slave laser will respond in a similar manner as the master laser, only it will do it at time $\tau_{c}$ while the master laser will do it at time $\tau$. The simultaneous turn-on of the lasers allows an understanding of the mechanism of anticipated synchronization, but the lasers also synchronize if they are turned on independently. The lower the optical coupling, the larger the transient time before the slave laser synchronizes to the master laser.

In Fig. 1(c) there are small parameter mismatches between the lasers, and a small amount of noise. The lasers are not perfectly synchronized, and, because $j_{2}$ is slightly lower than $j_{1}, I_{1}\left(t+\tau-\tau_{c}\right)-I_{2}(t)$ fluctuates about a mean value different from zero. Bursts of desynchronization are observed when $I_{1}$ drops to zero.

Anticipated synchronization also occurs if the lasers operate at a higher injection current, but in this case $\gamma_{1}$ must be either moderately low $\left(\gamma_{1} \sim 2-3 \mathrm{~ns}^{-1}\right)$ or very high $\left(\gamma_{1}>40 \mathrm{~ns}^{-1}\right)$. For low optical feedback, $I_{1}$ exhibits destabilized relaxation oscillations and Fig. 1(d) shows that in this regime anticipated synchronization can be achieved. At a higher feedback level, the intensity fluctuations become larger, and frequent bursts of desynchronization are observed. At an even higher feedback, LFFs occur and synchronization can be achieved again. The study of the conditions for synchronization is in progress and will be reported elsewhere.

The degree of synchronization and the lag time between the lasers can be quantified by calculating the similarity function, defined as [21]

$$
S^{2}\left(\tau_{0}\right)=\frac{\left\langle\left[I_{1}\left(t+\tau_{0}\right)-I_{2}(t)\right]^{2}\right\rangle}{\left[\left\langle I_{1}(t)^{2}\right\rangle\left\langle I_{2}(t)^{2}\right\rangle\right]^{1 / 2}} .
$$

Figure 2(a) shows the similarity function when there is perfect anticipated synchronization. $S$ presents a sharp minimum at $\tau_{0}=\tau-\tau_{c}$. There are also additional minimums at $\tau_{0}=n \tau-\tau_{c}$ (with $n$ integer), which arise from time correlations of the master intensity. For larger injection current and low feedback, the shape of $S$ is qualitatively the same, but, in between the minimums, $S$ presents large oscillations at the relaxation-oscillation period, which are also due to correlations of $I_{1}(t)$.

When there is noise and parameter mismatches, $S\left(\tau_{0}\right)$ has a similar shape, but the minimums are less pronounced [see Fig. 2(b)]. The degree of synchronization is good, in a relatively large range of negative detunings, but typically synchronization is poor if $\Delta \omega>0$. This is an effect of the $\alpha$ factor that will be discussed elsewhere. If the lasers are subjected to slightly different injection currents, synchronization is good if $j_{1}>j_{2}$, and is relatively poor otherwise. In all cases studied, when the lasers are not identical, the best synchronization is achieved when the slave laser is a solitary laser.

Next, it is shown that anticipated and retarded synchronizations occur in time-delay systems in a one-way delayed coupling configuration. A partial replacement 

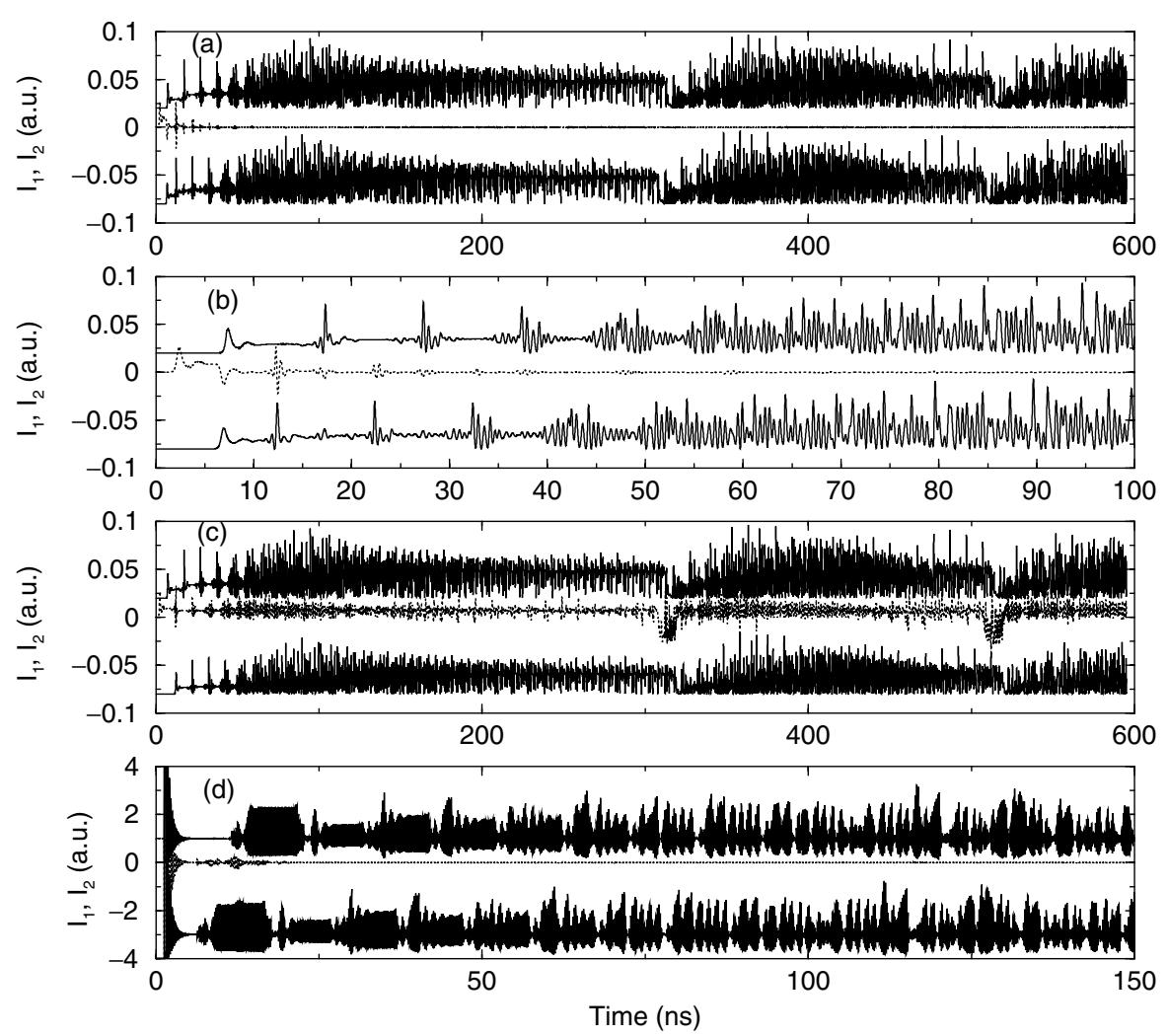

FIG. 1. Intensities of the lasers as a function of time $\left[I_{1}\right.$ and $I_{2}$ have been displaced vertically for clarity: $I_{1}\left(I_{2}\right)$ is the upper (lower) trace]. The dotted lines indicate the value of $I_{1}\left(t+\tau-\tau_{c}\right)-I_{2}(t) . \tau=10 \mathrm{~ns}$ and $\tau_{c}=5$ ns. (a) The lasers are identical and the noise level is zero. The parameters are $k=500 \mathrm{~ns}^{-1}, \epsilon=0.1, \tau_{n}=1 \mathrm{~ns}, \alpha=3, j=1.01, \omega \tau=3 \mathrm{rad}, \gamma_{1}=10 \mathrm{~ns}{ }^{-1}$, $\gamma_{2}=5 \mathrm{~ns}^{-1}, \eta=5 \mathrm{~ns}^{-1}$. (b) Simultaneous turn-on when the lasers are identical. The parameters are the same as in Fig. 1(a). (c) Same as Fig. 1(a), but the lasers have slightly different injection currents and optical frequencies $\left(j_{2}=1.0, \omega_{2}=0.299 \mathrm{GHz}\right)$, and the noise level is $\beta_{1}=\beta_{2}=0.01 \mathrm{~ns}^{-1}$. $\gamma_{2}=0 \mathrm{~ns}^{-1}$ and $\eta=10 \mathrm{~ns}^{-1}$ [all other parameters are the same as Fig. 1(a)]. (d) Anticipated synchronization for larger injection current. The lasers are identical and the noise level is zero. $j=2, \gamma_{1}=2.5 \mathrm{~ns}^{-1}$, $\gamma_{2}=0.25 \mathrm{~ns}^{-1}, \eta=2.25 \mathrm{~ns}^{-1}$, and $\epsilon=0.001$ [all other parameters are the same as Fig. 1(a)].

configuration is considered, in which the delayed function of the slave system, $g\left(y_{\tau}\right)=g(y(t-\tau))$, is partially replaced by the delayed function of the master system, $g\left(x_{\tau_{c}}\right)=g\left(x\left(t-\tau_{c}\right)\right)$,

$$
d x / d t=f(x)+\gamma_{1} g\left(x_{\tau}\right)+\beta_{1} \xi_{1}(t),
$$

$$
d y / d t=f(y)+\gamma_{2} g\left(y_{\tau}\right)+\eta g\left(x_{\tau_{c}}\right)+\beta_{2} \xi_{2}(t) .
$$

In (4) and (5), $\xi_{1}, \xi_{2}$ are independent Gaussian white noises of zero mean, and $\beta_{1}, \beta_{2}$ measure the noise in- tensity. Figure 3 shows simulations of coupled MackeyGlass equations [22] $\left[f(x)=-b x, g\left(x_{\tau}\right)=a x_{\tau} /(1+\right.$ $\left.x_{\tau}^{10}\right)$, Fig. 3(a)], and coupled Ikeda equations [23] [ $f(x)=$ $-b x, g\left(x_{\tau}\right)=a \sin \left(x_{\tau}\right)$, Fig. 3(b)]. In both cases the slave anticipates the chaotic master, in spite of the fact that the value of the parameter $b$ is slightly different for the master and slave systems, and there is a small amount of noise. $S\left(\tau_{0}\right)$ presents a single minimum at $\tau_{0}=\tau-\tau_{c}$, because no time correlations exist in the signal $x(t)$.
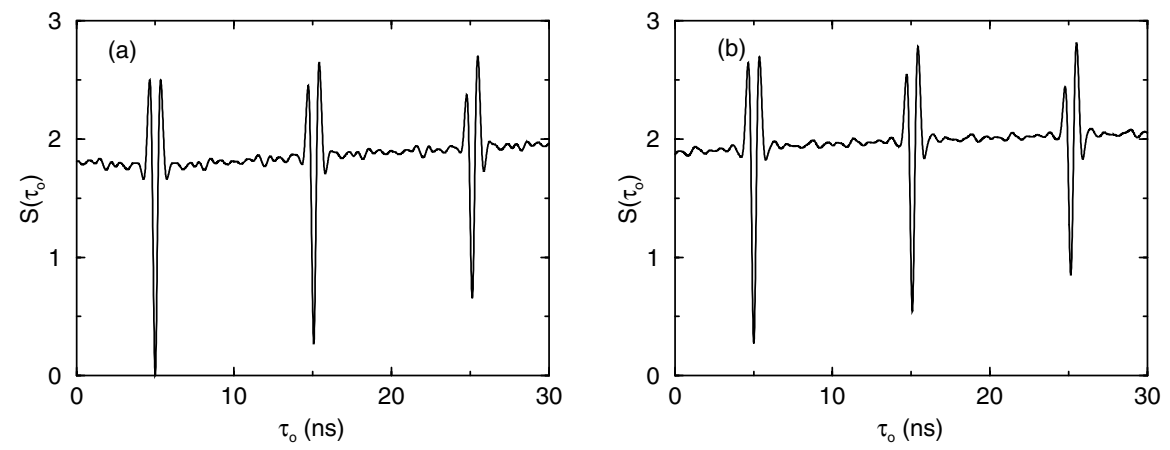

FIG. 2. Similarity function (a) for the same parameters as Fig. 1(a), and (b) for the same parameters as Fig. 1(c). $S\left(\tau_{0}\right)$ was calculated, averaging over 100 time series with different noise realizations. 

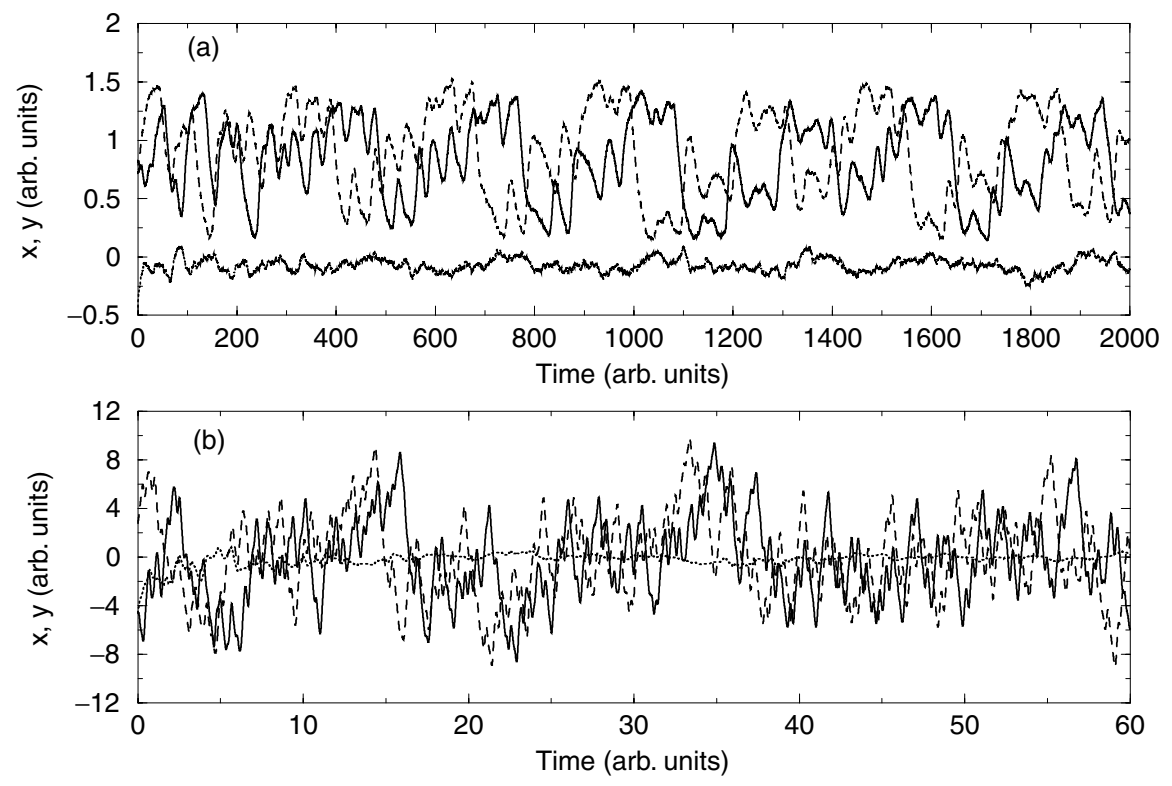

FIG. 3. (a) Simulations of coupled Mackey-Glass equations with $a_{1}=a_{2}=0.2, b_{1}=0.1, b_{2}=0.09, \beta_{1}=\beta_{2}=0.01, \tau=100$, $\tau_{c}=10, \gamma_{1}=1, \gamma_{2}=0.2$, and $\eta=0.8$. (b) Simulations of coupled Ikeda equations with $a_{1}=a_{2}=20, b_{1}=1, b_{2}=0.9$, $\beta_{1}=\beta_{2}=0.01, \tau=2, \tau_{c}=0.5, \gamma_{1}=1, \gamma_{2}=0.2$, and $\eta=0.8$. In both (a) and (b), the solid lines represent $x(t)$, the dashed lines represent $y(t)$, and the dotted lines represent $x\left(t+\tau-\tau_{c}\right)-y(t)$.

In summary, the synchronization of two chaotic semiconductor lasers with optical feedback was studied numerically, and a regime of anticipated synchronization was found. In this regime, which occurs when the coupling is large enough, $\tau>\tau_{c}$, and $\gamma_{1}=\gamma_{2}+\eta$, the slave laser anticipates the chaotic output of the master laser. It was also shown that this regime occurs generically in unidirectionally coupled delay-differential equations, when the coupling is set delayed in time, such that $\tau>\tau_{c}$.

The simulations suggest that anticipation is robust to small noise and parameter mismatches. It would be interesting to search for it in experiments with optically coupled chaotic external-cavity lasers, and with MackeyGlass-type electronic circuits. The signature of anticipation (a pronounced minimum at $\tau_{0}=\tau-\tau_{c}$ in the similarity function) might be found in experiments, where, due to unavoidable noise and parameter mismatches, complete synchronization is very difficult to observe.

The author gratefully acknowledges lively and stimulating discussions with Professor J. R. Rios Leite, and wishes to thank Professor J. Ohtsubo for his comments on the experimental observations. This work was supported by PEDECIBA and CSIC.

[1] H. Fujisaka and T. Yamada, Prog. Theor. Phys. 69, 32 (1983).

[2] L. M. Pecora and T. L. Carroll, Phys. Rev. Lett. 64, 821 (1990).

[3] S. Hayes et al., Phys. Rev. Lett. 70, 3031 (1993).

[4] K. M. Cuomo and A. V. Oppenheim, Phys. Rev. Lett. 71, 65 (1993).
[5] Y. Liu et al., Phys. Rev. A 50, 3464 (1994).

[6] G. D. VanWiggeren and R. Roy, Science 279, 1200 (1998); Phys. Rev. Lett. 81, 3547 (1998).

[7] J.-P. Goedgebuer et al., Phys. Rev. Lett. 80, 2249 (1998).

[8] G. Perez and H. A. Cerdeira, Phys. Rev. Lett. 74, 1970 (1995).

[9] K. M. Short, Int. J. Bifurcation Chaos Appl. Sci. Eng. 4, 957 (1994); Int. J. Bifurcation Chaos 6, 367 (1996); K. M. Short and A. T. Parker, Phys. Rev. E 58, 1159 (1998); J. B. Geddes et al., Phys. Rev. Lett. 83, 5389 (1999).

[10] K. Petermann, IEEE J. Sel. Top. Quantum Electron. 1, 480 (1995); G.H.M. Van Tartwijk and D. Lenstra, Quantum Semiclass. Opt. 7, 87 (1995); C. Masoller and N. B. Abraham, Phys. Rev. A 57, 1313 (1998).

[11] S. Sivaprakasam and K. A. Shore, Opt. Lett. 24, 1200 (1999); IEEE J. Quantum Electron. 36, 35 (2000).

[12] Y. Takiguchi et al., Opt. Lett. 24, 1570 (1999); H. Fujino and J. Ohtsubo, Opt. Lett. 25, 625 (2000).

[13] I. Fischer et al., Phys. Rev. A 62, 011801-1R (2000).

[14] C. R. Mirasso et al., IEEE Photonics Technol. Lett. 8, 299 (1996).

[15] V. Ahlers et al., Phys. Rev. E 58, 7208 (1998).

[16] P. S. Spencer and C. R. Misasso, IEEE J. Quantum Electron. 35, 803 (1999).

[17] J. K. White and J. V. Moloney, Phys. Rev. A 59, 2422 (1999).

[18] H. U. Voss, Phys. Rev. E 61, 5115 (2000).

[19] R. Lang and K. Kobayashi, IEEE J. Quantum Electron. QE-16, 347 (1980).

[20] I. Fischer et al., Phys. Rev. Lett. 76, 220 (1996); G. Vaschenko et al., Phys. Rev. Lett. 81, 5536 (1998); J. Hales et al., Phys. Rev. Lett. 85, 78 (2000).

[21] M. G. Rosenblum et al., Phys. Rev. Lett. 78, 4193 (1997).

[22] M. C. Mackey and L. Glass, Science 197, 287 (1977).

[23] K. Ikeda, Opt. Commun. 30, 257 (1979). 